\title{
Autologous Adult Cortical Cell Transplantation Enhances Functional Recovery Following Unilateral Lesion of Motor Cortex in Primates: A Pilot Study
}

\author{
Mélanie Kaeser, $\mathrm{PhD}^{*} \ddagger \S$ \\ Jean-François Brunet, PhD*§ \\ Alexander Wyss, $\mathrm{PhD} \ddagger$ \\ Abderraouf Belhaj-Saif, PhD $\ddagger$ \\ Yu Liu, PhD $\ddagger$ \\ Adjia Hamadjida, MSc $\ddagger$ \\ Eric M Rouiller, $\mathrm{PhD} \ddagger \Phi$ \\ Jocelyne Bloch, MD§ণ \\ \#Unit of Physiology, Department of \\ Medicine, University of Fribourg, Fri- \\ bourg, Switzerland; §Department of \\ Neurosurgery, Lausanne University Hos- \\ pital (CHUV), Lausanne, Switzerland \\ * $₫$ The authors contributed to this article \\ equally.

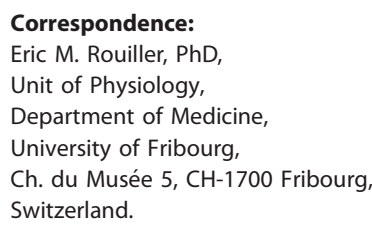 \\ E-mail: Eric Rouiller@unifr.ch
}

BACKGROUND: Although cell therapy is a promising approach after cerebral cortex lesion, few studies assess quantitatively its behavioral gain in nonhuman primates. Furthermore, implantations of fetal grafts of exogenous stem cells are limited by safety and ethical issues.

OBJECTIVE: To test in nonhuman primates the transplantation of autologous adult neural progenitor cortical cells with assessment of functional outcome.

METHODS: Seven adult macaque monkeys were trained to perform a manual dexterity task, before the hand representation in motor cortex was chemically lesioned unilaterally. Five monkeys were used as control, compared with 2 monkeys subjected to different autologous cells transplantation protocols performed at different time intervals.

RESULTS: After lesion, there was a complete loss of manual dexterity in the contralesional hand. The 5 "control" monkeys recovered progressively and spontaneously part of their manual dexterity, reaching a unique and definitive plateau of recovery, ranging from $38 \%$ to $98 \%$ of prelesion score after 10 to 120 days. The 2 "treated" monkeys reached a first spontaneous recovery plateau at about 25 and 40 days postlesion, representing $35 \%$ and $61 \%$ of the prelesion performance, respectively. In contrast to the controls, a second recovery plateau took place 2 to 3 months after cell transplantation, corresponding to an additional enhancement of functional recovery, representing $24 \%$ and $37 \%$ improvement, respectively.

CONCLUSIONS: These pilot data, derived from 2 monkeys treated differently, suggest that, in the present experimental conditions, autologous adult brain progenitor cell transplantation in a nonhuman primate is safe and promotes enhancement of functional recovery.

KEY WORDS: Adult, Autologous, Lesion, Monkey, Motor cortex, Neurotransplantation, Recovery
B rain lesions in the adult have dramatic consequences, because the spontaneous capacity of the brain to functionally recover is limited. Besides existing rehabilitative therapeutic approaches (eg, physiotherapy), several lines of research aim at developing treatments to promote and refine brain plasticity to enhance functional recovery following brain injury. Stem cell research, which represents

ABBREVIATIONS: M1, primary motor cortex; PM, premotor cortex; SGZ, subgranular zone; SVZ, subventricular zone a promising strategy to treat several nervous diseases, segregates into 2 main potential lines of treatment: implantation of exogenous cells or use of endogenous cells. The implantation of exogenous neural stem cells, either of embryonic or fetal origin, represents an encouraging approach, although there are serious limitations on the ethical, scientific, and clinical viewpoint. ${ }^{1-12}$ Another strategy aims at stimulating and/or recruiting endogenous stem cells or precursor cells available in the adult central nervous system. These cells are located principally in the forebrain subventricular zone (SVZ) and in the subgranular zone (SGZ) of the dentate gyrus of 
the hippocampus. ${ }^{12}$ These cells, however, migrate either only to a specific target (olfactory bulb from the SVZ) or only locally in the dentate gyrus (from the SGZ). The possibility to repair postlesion parts of the central nervous system (cerebral cortex, basal ganglia, spinal cord) remote from these 2 neurogenic niches is therefore limited. Attempts to experimentally enhance the migration of stem/precursors from the neurogenic niches to distant zones of the brain or to use resident progenitors led to a modest compensatory replacement of lost cells, without clinically relevant functional recovery. ${ }^{12}$ Stem/precursors taken from the SVZ or SGZ and transplanted into another brain area may not develop their full potential of cell replacement in a different environment. ${ }^{12}$ Stem/precursors residing in the cerebral cortex or basal ganglia may be too few to successfully replace lost cells after a lesion. To bypass these 2 limitations, the aim of the present pilot study was to extend preliminary experiments to fully test the feasibility and functional relevance of an original endogenous strategy in a nonhuman primate model of cerebral (motor) cortex lesion: first, to collect adult neural progenitor cells resident in the cerebral cortex of the lesioned subject itself ${ }^{13}$; second, to put the progenitor cells in culture to increase their number ${ }^{14,15}$; and third, to transplant them into the cerebral cortex, in and/or nearby an experimental lesion and assess the functional outcome.

A crucial step toward feasible, safe, and efficient clinical application requires the most appropriate animal model for the corresponding neural pathology, closely mimicking the characteristics of human diseases. The nonhuman primate model is often mandatory to address safety issues and scientific concerns, especially to include exquisite neural functions present only in primates. ${ }^{16,17}$ In a previous study, we established in 3 monkeys the basis for feasible autologous cell reimplantation, from the point of view of safety and the fate of the implanted cells. ${ }^{15}$ As a next step, the present pilot study aimed at providing, as proof of principle, first quantitative behavioral evidence in macaque monkeys for a beneficial outcome of cell therapy following injury of the cerebral cortex. To this aim, we used a previously established model of cerebral cortex lesion in nonhuman primates, ${ }^{18}$ introduced originally to assess the extent and time course of "spontaneous" functional recovery. ${ }^{19,20}$

\section{MATERIALS AND METHODS}

\section{Subjects}

Data were collected from 7 adult macaque monkeys (Macaca fascicularis; 6 males and 1 female) weighing between 3 and $6 \mathrm{~kg}$, ranging from 2.5 to 5 years old at onset of motor training sessions. The monkeys originated from the breeding colony in our own animal facility or were purchased from a certified supplier (Bioprim; 31450 Baziège; France), with the authorization to import delivered by the Federal Veterinary Office (BVET, Bern, Switzerland). All procedures were conducted in accordance to the Guide for Care and Use of Laboratory Animals (ISBN 0-309-05377-3; 1996) and approved by local veterinary authorities. Separate data from monkeys included in the present study were reported in previous articles or abstracts on other parameters. ${ }^{18,21,22}$
Seven adult macaque monkeys were subjected to unilateral lesion of the hand representation in motor cortex, producing a selective paresis of the contralesional hand. ${ }^{18}$ Postlesion, 2 of these monkeys were "treated" (autologous adult brain progenitor cells were reimplanted) for comparison of their functional recovery of manual dexterity with 5 lesioned, untreated ("control") monkeys.

Monkeys were housed in our animal facilities in rooms of $12 \mathrm{~m}^{3}$, in which usually 2 to 4 monkeys were free to move and interact with each other (a new swiss regulation has been introduced in September 2010 requesting a new volume of $45 \mathrm{~m}^{3}$ at least to be given to a group of 2-5 macaque monkeys). Before behavioral testing in the morning, the animal caretaker placed the monkeys in temporary cages for subsequent transfer to a primate chair. The monkeys had free access to water and were not food deprived. The rewards (pellets) obtained during the behavioral tests represented the first daily access to food. After the tests, the monkeys received additional food (fruits, cereals). The body weight of the animals was monitored before every behavioral session. In case the body weight dropped by $10 \%$ or more, the experiment was interrupted until the monkey regained the lost weight (this criterion for interruption was not met in the course of the present experiments).

\section{Behavioral Task}

The seven monkeys were trained to perform the modified (static) Brinkman board task, aimed at assessing manual dexterity. ${ }^{18-25}$ The subjects had to grasp food pellets from small slots oriented either vertically $(n=25)$ or horizontally $(n=25)$ by using precision grip (opposition of thumb and index finger), as described earlier in detail. ${ }^{23}$ Each monkey was its own control, as the dexterity scores were compared prevs postlesion. The slot dimension was $15 \mathrm{~mm}$ long, $8 \mathrm{~mm}$ wide, and 6 $\mathrm{mm}$ deep. The monkeys performed the task daily with each hand until they reached a plateau reflecting a stable prelesion performance. The duration of the daily behavioral session was about 1 hour. After the motor cortex lesion, the behavioral sessions were pursued for a period ranging from 80 to 310 days. The main analysis was focused on the number of pellets retrieved in 30 seconds (retrieval score) from the vertical and the horizontal slots, respectively. The total retrieval score was also documented (the sum of the pellets retrieved in 30 seconds from both slot orientations). Besides the retrieval score, reflecting the entire motor sequence (reaching, grasping, and withdrawal of the hand), a second parameter was measured, namely the contact time, as recently reported. ${ }^{2,23,26}$ The contact time is defined as the time of contact (in seconds) between the fingers and the pellet, representing the time interval between the first contact with the pellet in the slot (usually with the index finger) and the precise time of successful grasping when the pellet is removed from the slot. For each session, the contact time was determined separately for each of the first 5 vertical slots and each of the first 5 horizontal slots visited by the monkey. In general, the contact time was longer for the horizontal than the vertical slots, in line with the notion that grasping is somewhat more difficult for the horizontal slots, thus requiring a longer manipulation of the pellet before successive grasping. ${ }^{23}$

In addition to the modified (static) Brinkman board task, another task was introduced to test the ability to grasp moving objects (pellets), presented on a circular Brinkman board rotating at a speed of 10 rotations per minute. The monkey had to anticipate the rotation of the Brinkman board, turning either clockwise or counterclockwise. In a given behavioral session, the monkeys performed, one hand after the other, the rotating Brinkman board task once for each direction of rotation, corresponding each to the prehension of 32 pellets in slots distributed in 4 concentric circles. The contact time was measured as 
described above in the modified Brinkman board, for the first 10 slots aimed by the monkey. The 2 grasping tasks (modified Brinkman board and rotating Brinkman board) can be seen on the following web page: http://www.unifr.ch/neuro/rouiller/research/motorcontcadre.htm.

\section{Animal Care for Surgery}

After completion of prelesion training, the animals were subjected to the following surgical procedures. The monkeys were first tranquilized with ketamine (Ketalar; Parke-Davis, $5 \mathrm{mg} / \mathrm{kg}$, intramuscularly); atropine was injected $(0.05 \mathrm{mg} / \mathrm{kg}$, intramuscularly) to reduce bronchial secretions. Before surgery, the animals were treated with the analgesic carprofen (Rymadil, $4 \mathrm{mg} / \mathrm{kg}$, subcutaneously) and the antibiotic Albipen (ampicillin 10\%, $30 \mathrm{mg} / \mathrm{kg}$, subcutaneously). Subsequently, they were anesthetized with intravenous perfusion of $1 \%$ propofol (Fresenius) mixed with a 5\% glucose solution (1 volume of propofol and 2 volumes of glucose solution); ketamine was added to the perfusion solution $(65 \mathrm{mg} / 100 \mathrm{~mL})$. To prevent edema, methylprednisolone (Solu-medrol, Pfizer) was added to the propofol/glucose solution $(1 \mathrm{mg} / \mathrm{mL})$. The level of anesthesia was kept at an optimal level with a perfusion rate of the propofol/glucose mixture of $0.1 \mathrm{~mL} \cdot \mathrm{min}^{-1} \cdot \mathrm{kg}^{-1}$. All surgeries were performed under sterile conditions. Heart rate, respiration rate, expired $\mathrm{CO}_{2}$, arterial $\mathrm{O}_{2}$ saturation, and rectal temperature were monitored throughout the surgery. Each monkey was chronically implanted over the left forelimb area in the primary motor cortex (M1) with a stainless steel recording chamber $(22 \times 17 \times 15 \mathrm{~mm})$ allowing intracortical microstimulation sessions to map the primary motor cortex and locate the hand representation, as previously described. ${ }^{18,27}$ After surgery, the monkeys received carprofen (pills of Rymadil mixed with food) daily and Albipen (subcutaneously) every 2 days for 1 to 2 weeks.

\section{Biopsy of Prefrontal Cortical Tissue and Cell Preparation}

In the 2 treated monkeys (Mk-JO and Mk-JA; during prelesion phase), following the same surgery protocol as described above, a squared bony sector of $8 \times 8 \mathrm{~mm}$ was opened above the right dorsolateral prefrontal cortex. The dura mater was incised and an approximate volume of 8 to $20 \mathrm{~mm}^{3}$ of cortical tissue was extracted using a surgical blade (no. 11, Paragon) and placed into sterile cold culture medium. ${ }^{15}$ As previously described, ${ }^{15,28}$ a few hours after the biopsy was performed, the cortical tissue collected was dissected to obtain gray matter cells, placed first in RPMI 1640 medium with fetal bovine serum and an antibiotic/ antimycotic cocktail under horizontal agitation, and then kept in culture medium without serum, in suspension and under slow agitation. There was no specific cell sorting other than a selection imposed by the medium. Before reimplantation, a batch of cells were stained for subsequent tracking with fluorescent viable membrane dyes, PKH26 (red) for the cells reimplanted into the lesion site (Mk-JO and Mk-JA) and with PKH67 (green) for another batch of cells, reimplanted near the lesion site in the intact cortex (only Mk-JA). For the reimplantation (see below), the cells were cryopreserved, as previously reported. ${ }^{14}$

After the biopsy specimen was extracted, the bony sector was put back in place on the dura mater and sewed to the skull. The muscle and skin were then sutured. The extraction of brain tissue biopsy from the prefrontal cortex did not affect the motor capacity to grasp the pellets from the Brinkman board tasks.

\section{Primary Motor Cortex Lesion}

Several weeks after the biopsy, the hand area in the left M1 was permanently lesioned in the 7 monkeys by multiple injections of ibotenic acid (either Fluka 99\% or Sigma 95\%), as previously reported. ${ }^{18,22}$ A volume of 1 to $1.5 \mu \mathrm{L}$ of ibotenic acid solution $(10 \mu \mathrm{g} / \mu \mathrm{L}$ in phosphate-buffered saline) was injected at each site by using a Hamilton microsyringe positioned at relevant sites previously defined by intracortical microstimulation. The total volume of ibotenic acid injected in each monkey ranged from 15 to $40 \mu \mathrm{L}$. More relevant than the volume of ibotenic acid injected is the volume of the actual lesion (volume of gray matter in motor cortex affected by the lesion), as determined for each monkey based on reconstruction of the lesion from consecutive histological sections stained for SMI-32. A typical lesion produced by infusion of ibotenic acid in M1 is illustrated in a recent report. ${ }^{22}$

\section{Cell Reimplantation}

Following the same surgical protocol as described above, cells contained in culture medium were reimplanted stereotaxically with a Hamilton microsyringe. For one monkey (Mk-JO), the reimplantation was performed into the lesion site 15 days after the motor cortex lesion. A total volume of $20 \mu \mathrm{L}$ with PKH26 labeled cells containing 12500 cells/ $\mu \mathrm{L}$ was injected. For the other monkey (Mk-JA), the first reimplantation was performed into the lesion site (PKH26-labeled cells) and near the lesion site in the intact cortex (PKH67-labeled cells), once the monkey had reached a spontaneous functional recovery plateau. A second reimplantation was performed in the intact cortex, near the lesion site 49 days later (PKH67-labeled cells). The total volume (concentration of 3000 cells $/ \mu \mathrm{L}$ ) injected was $100 \mu \mathrm{L}$ with PKH26 cells and $100 \mu \mathrm{L}$ with PKH67-labeled cells (first implantation) and $49 \mu \mathrm{L}$ with PKH67labeled cells (second implantation), respectively. The 2 distinct viable membrane markers aimed at distinguishing, in the histological analysis, cells implanted in the lesion site from cells implanted in the intact cortex adjacent to the lesion (in Mk-JA).

\section{Necropsy and Histology}

At the end of the experiment, the monkeys were sacrificed as previously described, ${ }^{27,29}$ for histological analysis of the lesion and of the implanted cells' fate. Frozen sections of the brain were cut in the frontal plane at a thickness of $50 \mu \mathrm{m}$, collected in 8 series for further processing to visualize different markers, such as SMI-32, glial fibrillary acidic protein, nestin, MAP2, and doublecortin, as previously reported. ${ }^{15,29}$ Standard light, fluorescent, and confocal microscope analyses were conducted. Sectors of the brain sections where PKH26- and PKH67-labeled cells had been detected were selected and anatomically analyzed further.

\section{RESULTS}

\section{Modified Brinkman Board}

The description of the results is focused on the total score (sum of vertical and horizontal slots). Before lesion, the monkeys exhibited stable scores of manual performance (Figure 1; see http:// www.unifr.ch/neuro/rouiller/ACCI/videos.htm video sequences 1, 5, and 9 for Mk-JO, Mk-JA, and Mk-GE, respectively). As a result of unilateral lesion of motor cortex, a total loss of the contralesional hand performance was observed, as reflected by a grasping score dropping to zero in the modified Brinkman board task, both in control and treated monkeys (Figure 1A and B; see http://www.unifr.ch/neuro/rouiller/ACCI/videos.htm video sequences 2, 6, and 10 for Mk-JO, Mk-JA, and Mk-GE, 

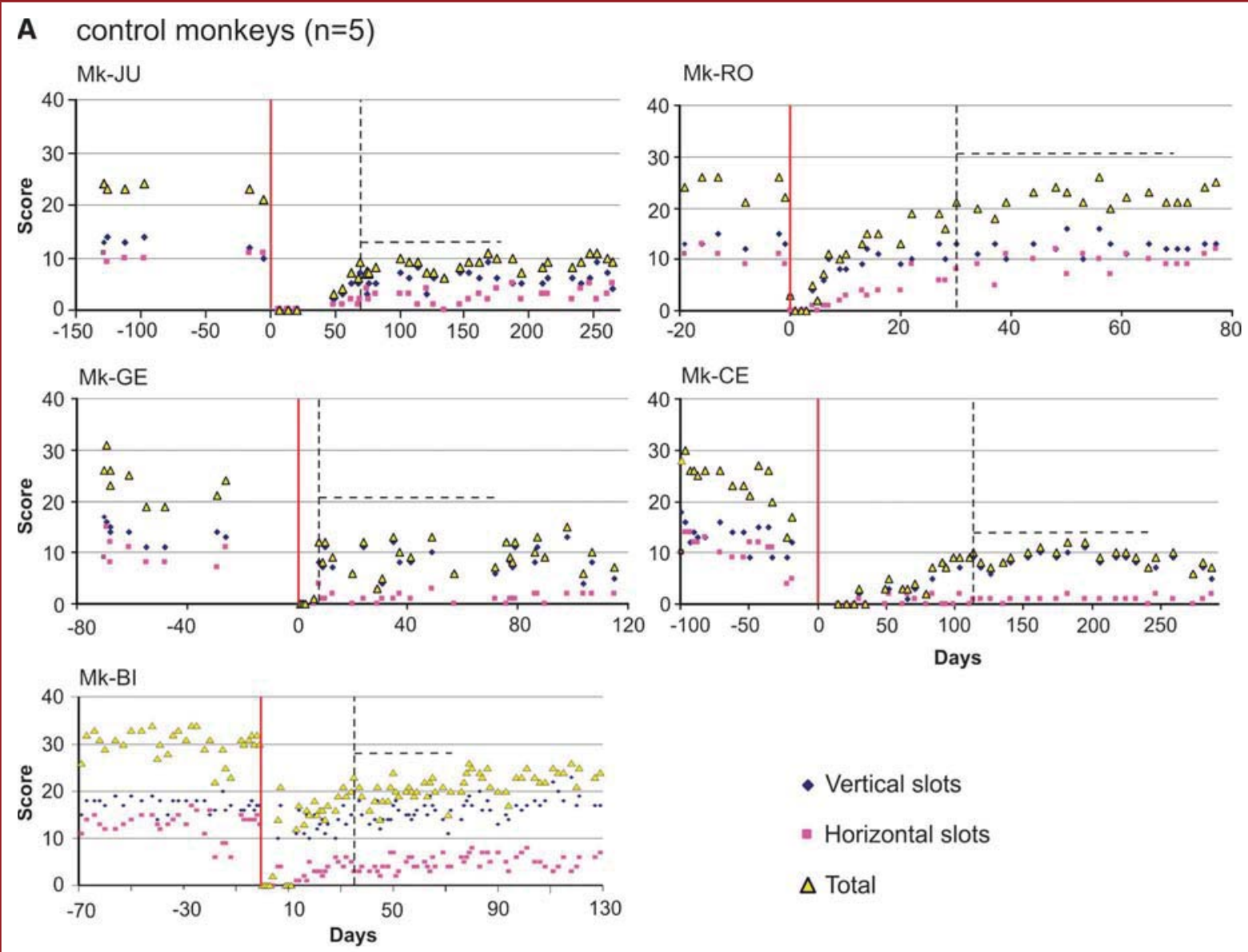

\section{- Vertical slots \\ - Horizontal slots}

$\Delta$ Total

\section{B re-implanted monkeys $(n=2)$}
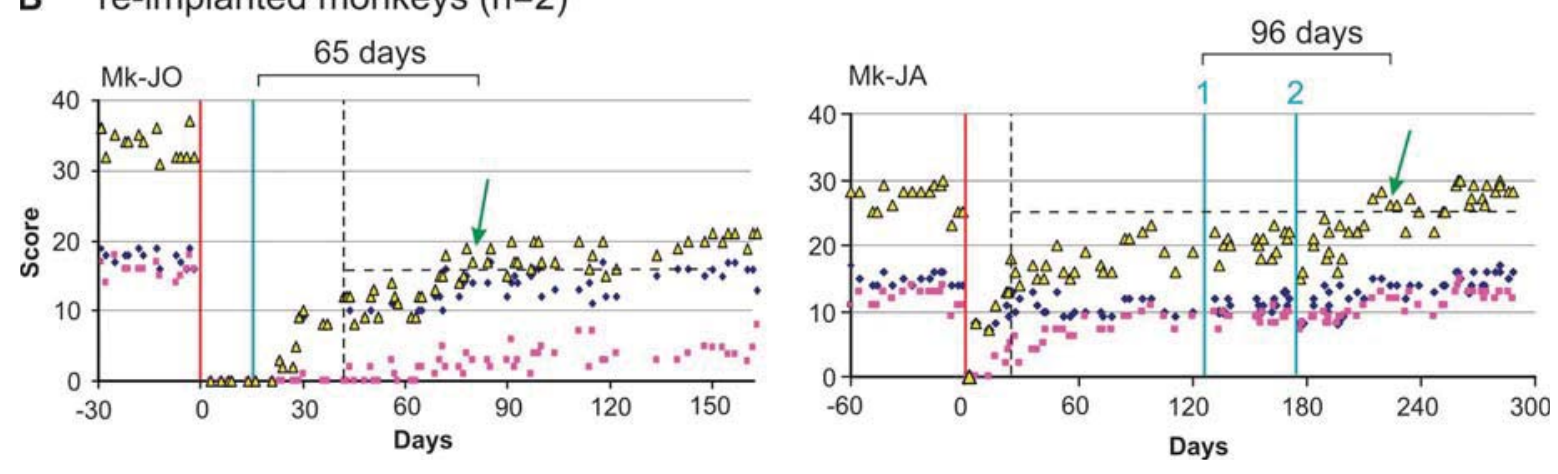

FIGURE 1. Behavioral data (retrieval score). A and $\mathbf{B}$, time course in days of manual dexterity performance (score), pre-and postlesion, as derived from the modified Brinkman board task in 5 control monkeys (A) and in 2 treated monkeys subjected to cell transplantation (B). The score is given by the number of pellets successively retrieved by the monkeys during 30 seconds. Yellow triangles represent the total score, blue diamonds the score for vertical slots, and pink squares the score for horizontal slots. The vertical red line indicates the time of the lesion (day zero). In $\mathbf{A}$, the vertical dashed line shows the time point at which a spontaneous plateau of recovery was reached (see text for criterion to define the plateau). In $\mathbf{B}$, the vertical dashed line represents the first (spontaneous) platean of recovery. In the 2 treated monkeys (B), the vertical blue lines show the time of autologous cell implantations. The green arrows point out to the onset of a second behavioral plateau, reached following a rebound in the recovery curve (see text), occurring after a delay after cell implantation given in days above the graph. The horizontal dashed line represents a level of score defined by the median score calculated at the platean of spontaneous recovery plus twice the difference between the highest value and the median score. In the control monkeys, the length of the horizontal dashed line represents the time window in which the spontaneous recovery was assessed quantitatively at plateau. The score level represented by the horizontal dashed line was taken as the criterion to be met to define a second plateau, representing an enhancement of functional recovery, observed in the 2 treated monkeys but not in the 5 control monkeys. 
respectively). The 5 control monkeys (Figure 1A) then exhibited a progressive, spontaneous functional recovery starting after 5 to 50 days, reaching a postlesion plateau 10 to 120 days postlesion, depending on the monkey. In the recovery curve approaching saturation, the onset of the plateau was defined as the first individual data point (total score) for which, among the next 3 individual data points, none exhibits a higher score (vertical dashed line in Figure 1A). This spontaneous recovery plateau ranged across the 5 control monkeys from 38 to $98 \%$ of the prelesion score of manual performance (Figure 2, top), thus

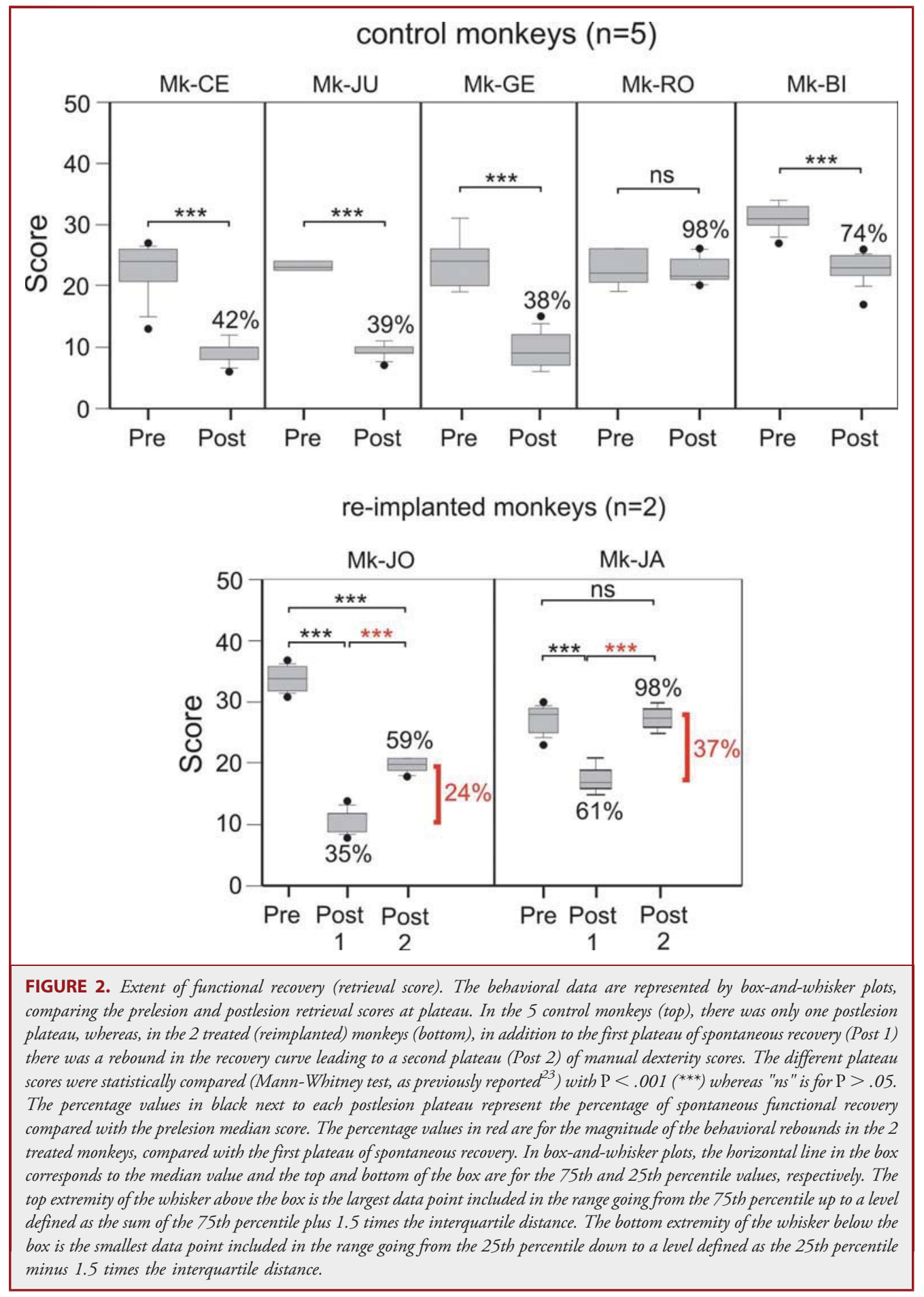


corresponding to an incomplete functional recovery. In the control monkey exhibiting the fastest recovery (Mk-GE), it turned out to be mainly limited to the vertical slots with a poor long-lasting recovery for horizontal slots (Figure 1A). A poor recovery for the horizontal slots was also observed in Mk-CE. The control monkey with the best recovery (Mk-RO, 98\%) had the smallest lesion. In Mk-RO, because the spontaneous recovery was nearly complete already after a couple of months, the experiment was interrupted after 80 days, in contrast to much longer periods of observation in the other monkeys. A statistical comparison of pre- and postlesion plateaux is presented in Figure 2 (top) for the 5 control monkeys, showing that the spontaneous recovery remained dramatically incomplete (38\% to $42 \%)$ in 3 of 5 monkeys. In the other 2 control monkeys, the spontaneous recovery was more prominent ( $74 \%$ to $98 \%)$, as expected for a smaller lesion of the motor cortex. The postlesion plateau then remained largely stable for several months in the control monkeys (Figure 1A).

For the 2 treated monkeys (Figure 1B), after complete loss of manual performance lasting 25 days in $\mathrm{Mk}-\mathrm{JO}$ and 5 days in Mk$\mathrm{JA}$, the same progressive spontaneous recovery as seen in control monkeys took place, reaching a first plateau 40 days after lesion in $\mathrm{Mk}-\mathrm{JO}$ and 25 days after lesion in Mk-JA (vertical dashed lines in Figure 1B). In Mk-JO, this first plateau represented $35 \%$ of prelesion score and lasted about 30 days (see http://www. unifr.ch/neuro/rouiller/ACCI/videos.htm video sequence 3). Meanwhile, 15 days after lesion, the autologous adult brain cell transplantation was performed. Starting around 70 days postlesion, there was a rebound of recovery, as reflected by a "second plateau" 80 days after the lesion (green arrow in Figure 1B), respectively, 65 days after the autologous transplantation. The second plateau was defined by data points in the recovery curve standing out above a level (horizontal dashed line in Figure 1B) calculated as the median score at first plateau to which twice the difference between the highest value and the median value at plateau 1 was added. The median score and the difference between the highest value and median were calculated from 15 data points taken from the beginning of the first plateau. The time onset of the second plateau was arbitrarily defined as the third data point (green arrow) greater than the limit (horizontal dashed line) defined above. This second behavioral plateau represented $59 \%$ of the prelesion score in Mk-JO. The difference between the first and second postlesion plateau (24\%) was statistically significant $(P<.001$; see red stars in Figure 2 ; see http:// www.unifr.ch/neuro/rouiller/ACCI/videos.htm video sequence 4 and compare with video sequence 3 at first plateau).

In the treated $\mathrm{Mk}-\mathrm{JO}$ described above, the autologous cell transplantation was performed into the lesioned territory in the motor cortex, 15 days after the lesion during the first recovery phase. In this monkey, as shown by the histological analysis, the reimplanted cells remained mainly at a precursor stage (positive for the markers nestin, vimentin, and doublecortin), surrounded by endogenous astrocytes (glial fibrillary acidic protein reactive gliosis), although some cells were found to be either engaged in a neuronal differentiation process (positive for the marker $\beta$-tubulin III) or differentiated (positive for the marker SMI-32). Because the transplanted cells were found to be agglomerated, they did not migrate at all to occupy the whole lesioned territory. In an earlier feasibility study without behavioral assessment, ${ }^{15}$ transplantation of autologous neural cells was performed in 3 monkeys not only in the lesion itself, but also in the adjacent intact tissue, near the lesioned territory, at a later time point after the lesion (40 days). The reimplanted cells showed in these 3 monkeys an ability to massively migrate from the reimplantation site toward the damaged area, as well as to differentiate massively (positive for the neuronal marker MAP-2). Based on these data (feasibility study and Mk-JO of the present pilot study), in the second monkey subjected here to the transplantation of autologous adult brain cells (Mk-JA), the reimplantation was performed both in and near the lesioned territory in M1, as well as at a later time point postlesion than in Mk-JO, to investigate the impact of these 2 parameters on the cell migration and differentiation ability.

In $\mathrm{Mk}-\mathrm{JA}$, the reimplantation of the autologous cells was performed in 2 steps, 125 days and 175 days postlesion. The first, spontaneous behavioral plateau represented $61 \%$ of the prelesion score and lasted more than 150 days (Figure 1; see http:// www.unifr.ch/neuro/rouiller/ACCI/videos.htm video sequence 7). Then, as in the other treated monkey, a rebound of recovery took place as reflected by a second plateau (data points above the horizontal dashed line in the right panel of Figure 1B) at about 96 days after the first implantation. Moreover, this second plateau exhibited further improvement at postlesion day 260, possibly in relation to the second implantation of autologous cells (corresponding to a delay of about 85 days). The second plateau corresponded to a nearly complete recovery (98\%): the enhancement of recovery presumably related to the cell transplantation was $37 \%$ (see red stars in Figure 2 for statistical comparison of plateaux 1 and 2; see http://www.unifr.ch/neuro/rouiller/ACCI/videos.htm video sequence 8 and compare with video sequence 7 at first plateau).

In the 2 treated monkeys, a rebound of manual performance score was present, as reflected by the second plateau of functional recovery. In contrast, in the 5 control monkeys, when the first plateau of recovery was reached, variations of the score across daily sessions never reached the criterion defined by the median score plus twice the difference between the highest value and the median score (horizontal dashed lines in Figure 1A). Among the 5 control monkeys, one animal (Mk-BI) exhibited some improvement after the plateau of recovery was reached, but it did not fulfill the criterion of surpassing the median score plus twice the difference between the highest value and the median score; the final level of recovery reached by $\mathrm{Mk}-\mathrm{BI}$ was separated from the criterion level (horizontal dashed line in Figure 1A) by a score gap of half the difference between the median score and the highest value at plateau. The enhancement of manual performance observed in the 2 treated monkeys, corresponding to the second plateau, is illustrated qualitatively by video sequences (see http://www.unifr.ch/neuro/rouiller/ ACCI/videos.htmcompare sequences 3 and 4 for $\mathrm{Mk}-\mathrm{JO}$ as well as sequences 7 and 8 for Mk-JA), showing that the 2 monkeys achieved a better manual performance at the level of the second plateau than 
at the level of the first plateau. For comparison, video sequences taken at 2 remote time points along the unique plateau of spontaneous recovery in Mk-GE show that there was no enhancement of manual dexterity (http://www.unifr.ch/neuro/rouiller/ACCI/videos.htm, compare video sequences 11 and 12).

To further support the notion that the second plateau observed exclusively in the 2 treated monkeys corresponds to an enhancement of manual dexterity per se (better manipulation of pellets between the thumb and index finger), a further analysis was based on the contact time (see Materials and Methods). The contact time was determined in the sessions included in time windows corresponding to the first and second plateaux in MkJO (40-68 postlesion days and 9-164 postlesion days for plateau 1 and plateau 2, respectively) and in Mk-JA (80-180 postlesion days and 252-300 postlesion days for plateau 1 and plateau 2, respectively). The distribution of the contact times observed at plateau 1 and 2 is shown in the form of box-and-whisker plots in Figure 3A, separately for the vertical and horizontal slots, for each of the 2 treated monkeys. In $\mathrm{Mk}-\mathrm{JO}$, irrespective of the slot orientation, the contact times were significantly shorter at plateau 2 than at plateau 1 , reflecting an improvement of manual dexterity when the second plateau was reached (Figure 3A). This was also the case in $\mathrm{Mk}-\mathrm{JA}$ for the vertical slots, whereas there was no statistically significant difference for the horizontal slots, although the contact times were less variable at plateau 2 than at plateau 1 (Figure 3A). In agreement with the retrieval score data, the extent of decrease of median contact times going from plateau 1 to plateau 2 in Mk-JO (both slot orientations) and Mk-JA (vertical slots) ranged from 24 to $30 \%$, representing an enhancement of manual dexterity comparable to the improvement of performance reflected by the retrieval score (24-37\%; Figure 2).

\section{Rotating Brinkman Board Task}

A separate assessment was made of whether the 2 monkeys (MkJO; Mk-JA) subjected to transplantation of autologous adult cortical cells achieved a second level of recovery that reflected a significant improvement of manual dexterity over the first plateau. In this assessment, the contact times were measured in the rotating Brinkman board, comparing the sessions taken within the time windows corresponding to the 2 distinct plateaux (see above). As shown in Figure 3B, irrespective of the direction of rotation of the board, there was a statistically significant decrease of contact time going from postlesion plateau 1 to postlesion plateau 2, indicative of an enhanced manual dexterity. Comparing the median values, there was a decrease of contact time from plateau 1 to plateau 2 ranging from $42 \%$ to $57 \%$, thus even more pronounced than for the modified (static) Brinkman board task.

\section{Relationship Between Lesion Size and Recovery}

There was a substantial variation of postlesion recovery across monkeys (Figures 1 and 2). To assess the dependence on lesion size (see Figure $4 \mathrm{~B}$ ), the degree of functional recovery in the modified Brinkman board (total score) in percent was plotted as a function of lesion volume, corresponding to the extent of gray matter in motor cortex affected by the lesion (Figure 4A). As expected, a small lesion in a control monkey (Mk-RO) was followed by a nearly complete spontaneous functional recovery (98\%). A somewhat larger lesion in a second control monkey (Mk-BI) was followed by a reduced, though still prominent $(74 \%)$, spontaneous recovery. In contrast, still in control monkeys, above a lesion volume of about $40 \mathrm{~mm}^{3}$ and up to a volume of about $100 \mathrm{~mm}^{3}$, the extent of spontaneous recovery remained fairly constant near $40 \%$ (Figure 4A). The extent of spontaneous recovery across the control monkeys is quite variable and the functional recovery observed in the 2 treated monkeys is in the same range. Nevertheless, a difference between the 2 subgroups of monkeys is the presence of a rebound in the postlesion recovery curve in the 2 treated monkeys (second plateau of an extent represented by the vertical dashed line in Figure 4A), a phenomenon absent in the 5 control monkeys (see also Figures 1 and 2).

The 2 treated monkeys of the present pilot behavioral study, as well as the 3 monkeys of our previous report also subjected to autologous cell transplantation, ${ }^{15}$ did not exhibit any sign of pain or discomfort related to the autologous cell transplantation, indicating that this procedure does not produce undesired, secondary effect.

\section{Cell Analysis}

When looking at SMI-32-stained brain sections in motor cortex in an intact territory, there were darkly stained SMI-32 positive neurons in layer III and layer $\mathrm{V}$, both in control and treated monkeys (Figure 4C, panel c1). In contrast, in the lesion territory, there was no SMI-32 staining in the control monkeys as expected (Figure 4C, panel c2), whereas lightly stained SMI-32 positive neurons were present in the lesion territory of the treated monkeys (Figure 4C, panel c3). When considering more closely these SMI-32-positive cells located in the lesion site of the treated monkey (Figure 4C, panel c4), it appeared that they sometimes corresponded to PKH26 (red, designed by yellow arrows) and PKH67 (green, designed by white arrows) positive cells that were transplanted in the monkey's brain (Figure 4C, panels c5-c7). In a treated monkey, a 3D reconstruction of the motor cortical area shows the distribution of PKH26 (red)- and PKH67 (green)labeled cells (Figure 4C, panels c8-c10), implanted respectively in the lesion and at the vicinity of the lesion. Finally, PKH67positive cells were present in the lesion site, indicating that they migrated from the intact cortex toward the lesion territory. The proportion of surviving transplanted cells amounted to about one third on average, a proportion most likely consistent with a clinical effect. The histological observations did not reveal the presence of teratoma or tumor formation.

\section{DISCUSSION}

\section{Survey of the Data}

To the best of our knowledge, the present behavioral pilot study is the first report in nonhuman primates suggesting that transplantation of autologous adult brain progenitor cells leads to 


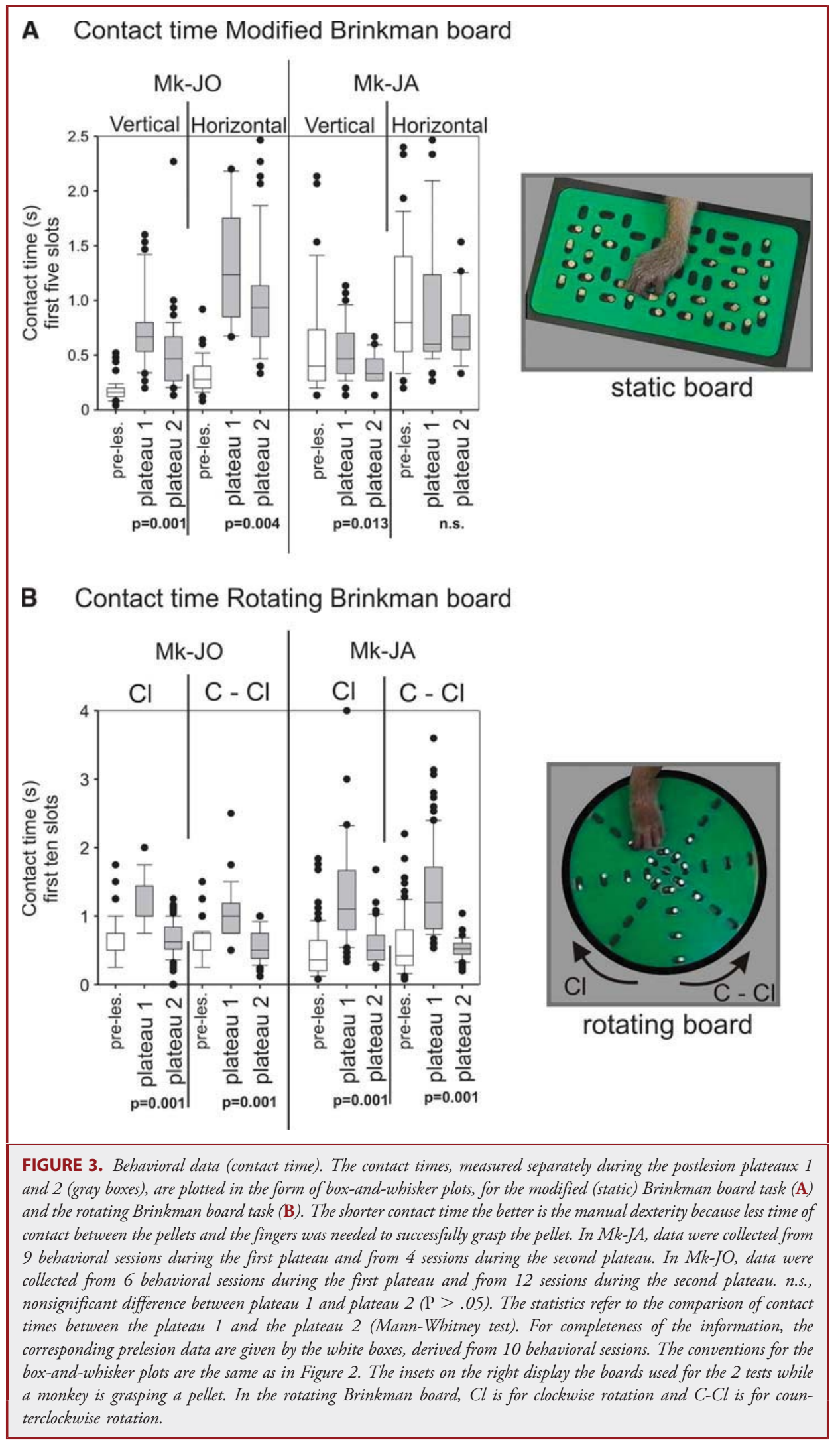


FIGURE 4. Relationship with morphological data. A, plot of the percentage of postlesion functional recovery of the contralesional hand (total score in the modified Brinkman board) as a function of the volume of the primary motor cortical lesion. Black-filled circles represent the control monkeys and blue-filled circles represent the monkeys treated with autologous brain cells. For the 2 treated monkeys, the vertical dashed line represents the additional recovery observed (rebound), presumably in relation to the cell reimplantation (see text). B, lateral views of the projection on the cerebral cortex surface of the approximate location and extent of the left MI gray matter lesion (red) as observed on frontal histological sections of the monkeys' brain. Theses reconstructions do not take the depth of the lesion into account. The precise extent of the lesion is given by its volume in $\mathbf{A}$. At the bottom, the brain of the 4 control monkeys are shown, whereas the brain of the 2 monkeys reimplanted with autologous brain cells are represented at the top. $A R$, arcuate sulci; $C E$, central sulci. Scale bar $=10 \mathrm{~mm} . \mathbf{C}$, histological data in transplanted and control lesioned motor cortex area. (C1 to C3) Brain diaminobenzidineimmunohistology for SMI-32 neurons. In C1, SMI-32positive neurons are as seen in the intact part of the motor cortex (at some distance from the lesion); SMI-32-positive neurons are present in layer III and layer V. The lesion territory is shown in $C 2$ and $C 3$ for a control monkey and a treated monkey, respectively; in the control monkey, no SMI-32 staining is visible (C2); in contrast, there are some positively stained cells in the lesion of the treated monkey (C3). Scale bar $=200 \mu \mathrm{m}$. (C4 to C7) High magnification of SMI-32-positive cells in the lesion site of the treated monkey (C4 and C7). These SMI-32positive cells (yellow and white arrows in C4 and C7) correspond to PKH26-labeled cells (yellow arrows in C5) or to PKH67-labeled cells (white arrows in C6). Scale bar $=200 \mu \mathrm{m} .(C 8-C 10) 3 D$ reconstruction of the motor cortex lesion territory showing the distribution of PKH67-labeled cells (green dots in C8 and C9) and PKH26-labeled cells (red dots in C9 and C10). Note that the 2 sets of cells migrated in the same lesion territory in the overlay reconstruction (C9). Scale bar $=1 \mathrm{~cm}$.

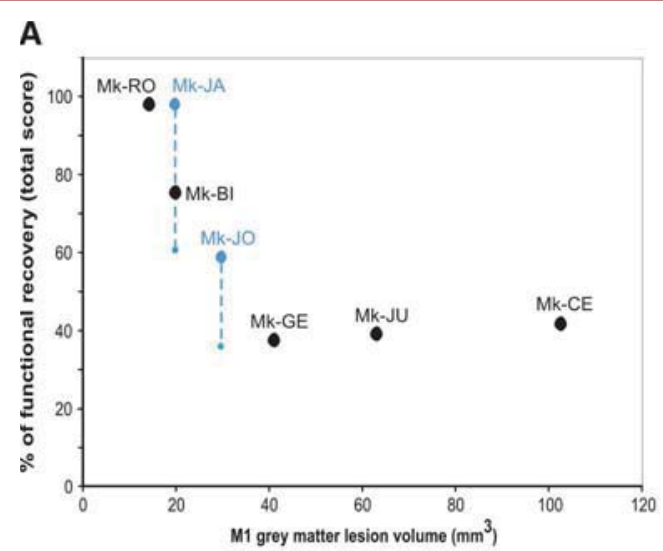

B
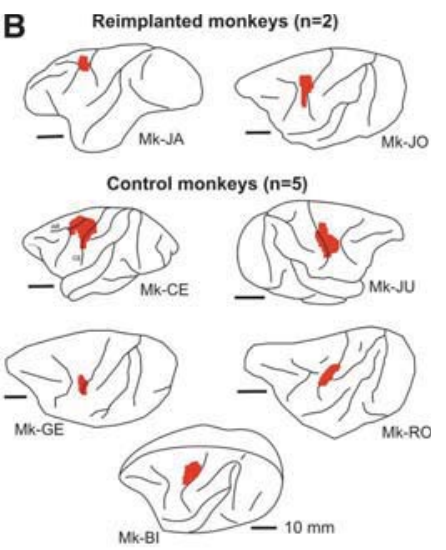

- Autologous neural progenitor cells treated monkeys - Control monkeys
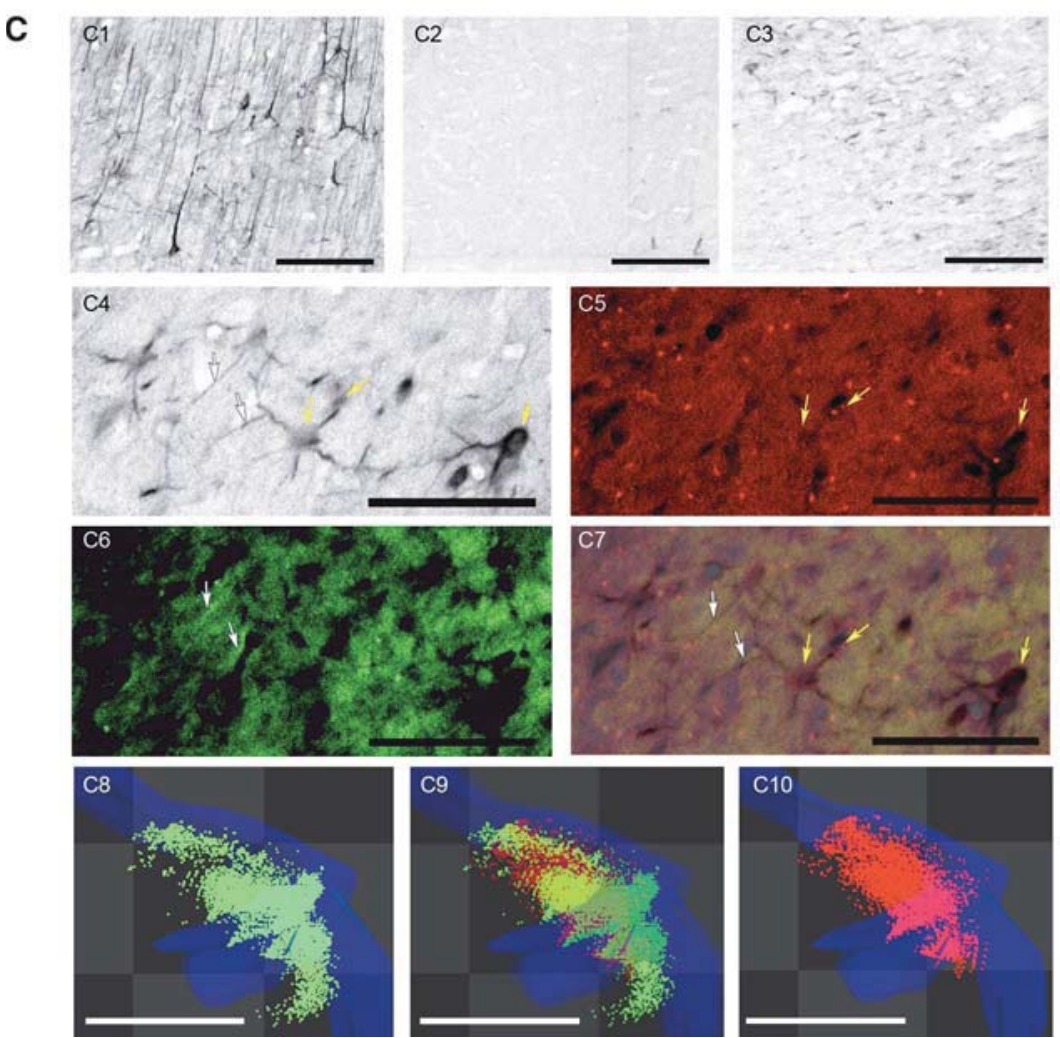

an enhancement of functional recovery after brain injury. The enhancement of recovery is not directly reflected by a difference of postlesion behavioral plateau between groups of "treated" and "control" monkeys: the recovery was quite variable across monkeys because of interindividual variability of lesion volume and precise position of the lesion. Because of this high variability, an intergroup comparison would require larger numbers of monkeys in each group. The preliminary evidence for enhancement of functional recovery in relation to the treatment is rather based on the presence of a rebound (second plateau) in the postlesion recovery curve, present in the 2 treated monkeys and not in the 5 control monkeys (Figures 1, 2, and 4). The manual performance obtained at the second plateau was higher than the performance reflected by the first plateau of recovery (Figures 1 , 
2, and 3; see also http://www.unifr.ch/neuro/rouiller/ACCI/ videos.htm video sequences). Furthermore, the rebounds observed in the treated monkeys turned out to occur within a fairly comparable time window in the 2 treated monkeys (about 2-3 months; Figure 1B). The enhanced functional recovery in the 2 treated monkeys (reflected by the second plateau), compared with the control monkeys, cannot be explained by a more frequent practice of the motor tasks postlesion in the monkeys subjected to the transplantation of autologous cells, because the frequency of behavioral sessions were overall comparable across the 2 groups of monkeys.

\section{Limitations of Interpretation}

The interpretation of the present pilot results is limited by the low number of monkeys, especially in the group of treated monkeys, limited to 2 cases. Nevertheless, the consistent data obtained in the 5 control monkeys in the sense of an absence of significant rebound of functional recovery (even after a long time postlesion; see Figure 1A) represents a reliable observation, considering that 5 monkeys constitute a decent group size for nonhuman primates, although a group of 6 to 7 animals would be optimal. ${ }^{23}$ More preliminary is the observation of a rebound of functional recovery most likely related to the autologous cell therapy, because it is based on only 2 monkeys, who underwent somewhat different injection protocols. However, the enhancement of recovery occurred with a time course consistent with plausible scenarios (see below). As far as the safety of the present therapeutic procedure is concerned, the 2 treated monkeys confirm the data previously obtained on 3 monkeys in a feasibility study. $^{15}$

\section{Possible Mechanisms of Enhancement of Functional Recovery}

The delay of 2 to 3 months between cell reimplantation and rebound of functional recovery may reflect 2 possible mechanisms: cell replacement and/or endogenous neurogenesis induced by factor delivery. First, the reimplanted cells may integrate the neuronal network after differentiation into mature neurons that express $\mathrm{MAP}^{15}$ and SMI-32 (Figure 4C). As shown previously, ${ }^{15} 1$ month after transplantation, the cells still expressed progenitor markers such as nestin and were differentiated into neurons at 3 months postreimplantation. Furthermore, Koch et $\mathrm{al}^{30}$ demonstrated that human embryonic neural stem cells can functionally integrate with and receive synaptic input from host brain tissue after 18 to 24 weeks. Such integration may occur in our study, where the autologous strategy is expected to facilitate the migration and integration of the transplanted cells. In line with this ability to colonize the entire lesion, the re-implanted cells express doublecortin, a migrant neuroblast marker that participates in the regulation of microtubule dynamics and stability during neuronal morphogenesis and migration. ${ }^{31}$ Second, the cells may have a bystander effect through factor delivery and influence the endogenous brain modeling. ${ }^{32}$ Preliminary results (data not shown) indicate that, in vitro, these adult primate brain cells secrete neurotrophic factors, such as brain derived neurotrophic factor, leukemia inhibitory factor, and ciliary neurotrophic factor. Based on the posttreatment delay of 2 to 3 months, the cell replacement mechanism appears likely but the factor delivery effect cannot be excluded at that time, although it should occur more rapidly. It is known that, after lesion of the M1 in monkey, the ipsilesional premotor cortex (PM) plays a significant role in the "spontaneous" functional recovery, ${ }^{18,33-35}$ although other cortical areas may contribute. ${ }^{36}$ Among possible mechanisms, it was shown that the normal projection from PM to M1 is redirected to the postcentral gyrus (somatosensory cortex $^{37}$ ). It may be that the transplanted autologous adult cortical cells secrete factors favorable for such redirection of relevant projections originating from intact adjacent motor cortical areas.

Both the absence of rebound in the recovery curve of the 5 control monkeys and the consistent delay of about 2 to 3 months between autologous cells implantation and the rebound leading to a second plateau argue in favor of a positive effect of the cell transplantation on the functional recovery of manual dexterity following lesion of the hand area in M1 in macaque monkeys. In line with such interpretation, the autologous cell implantation took place at different time points with respect to the lesion in the 2 treated monkeys. If the rebounds would be due to factors independent from the autologous cells' implantation, then a consistent delay of 2 to 3 months would most likely not have been observed in the 2 treated monkeys. Another important observation derived from $\mathrm{Mk}-\mathrm{JA}$ is that the rebound in the postlesion recovery curve occurred even though the autologous cell implantation took place a long time after the lesion (more than 3 months). The delay between the lesion and the cell reimplantation may play an important role, and the optimal timing remains to be determined in future experiments. For instance, it may be favorable to wait for the completion of the whole perilesional inflammatory phenomenon to obtain an optimal cell migration and differentiation.

The magnitude of the rebound of the postlesion recovery curve in the 2 treated monkeys ranged from 24 to $37 \%$, as assessed in the modified Brinkman board task, adding to the "spontaneous" postlesion recovery score (Figure 2). In the second behavioral test (rotating Brinkman board), the effect even reached about 50\% improvement of manual performance (Figure 3). In the control monkeys, there was no sham treatment (for instance, infusion of vehicle only). However, it is unlikely that the absence of rebound is due to the lack of cortical penetration in the perilesional territory with a syringe. In the entire cohort of monkeys with M1 lesion (not Mk-JO and Mk-JA, but comprising monkeys not included in the present study, eg, treated with anti-Nogo-A antibody), postlesion intracortical microstimulation sessions conducted at the level of the plateau of recovery did not exhibit a second plateau within the next 2 to 3 months, indicating that a surgical insult in itself does not enhance functional recovery.

A similar rebound effect as in the 2 monkeys with cell transplantation was found in another group of monkeys subjected to a comparable lesion of M1, but treated with anti-Nogo-A 
antibody, ${ }^{21}$ a therapeutic strategy known to also enhance functional recovery after cervical cord lesion in monkeys. ${ }^{23,38}$ The time course of rebound observed in the anti-Nogo-A antibodytreated monkeys subjected to lesion of M1 was comparable to the rebound delay of about 2 to 3 months observed here in the monkeys subjected to autologous transplantation. This observation suggests that both treatments, although based on different mechanisms, exert their effect following a comparable time course, which may represent a favorable situation to tentatively combine the 2 treatments in future experiments in monkeys, with the aim to obtain an additive, if not synergistic effect.

\section{Comparison With Previous Studies}

Following stroke, a majority of patients experience serious loss of hand function. The present monkey model focused on the recovery of hand function after cortical lesion thus represents a pertinent model to test therapeutic strategies. A direct comparison of the present data with previous animal studies is limited, because nearly all preclinical studies of cell therapy on animal models of cerebral infarct were conducted on rodents as the host animal. ${ }^{10}$ One exception is a recent study, ${ }^{39}$ in which human neural stem cells were transplanted in a monkey model of ischemic stroke. Evidence for cell replacement was found but the functional outcome was not tested. Considering another neurological disease (Parkinson disease), the transplantation of dopaminergic neurons generated from embryonic stem cells in monkeys into 1-methyl-4phenyl-1,2,3,6-tetrahydropyridine monkeys was followed by an attenuation of neurological symptoms, ${ }^{40}$ based on a neurological score testing a wide repertoire of functions, but less quantitative than the present assessment of manual dexterity. In a monkey model of Parkinson disease, using the same transplantation strategy of autologous adult progenitor cells, there is also evidence for a neuroprotective effect ${ }^{28}$ and enhancement of functional recovery compared with control monkeys. ${ }^{41}$ Taken together, the latter study on Parkinson disease and the present one on cortical lesion, both based on reimplantation of autologous adult progenitor cells, provide new and encouraging results, as suggested by an enhanced functional recovery possibly related to the treatment. However, these 2 studies are at a pilot step and therefore the conclusion of enhanced functional recovery prompted by autologous cell transplantation needs to be confirmed on a larger sample of monkeys and, preferably, in combination with other strategies, such as neutralization of Nogo-A, for instance. ${ }^{23,38}$ Even at the present pilot stage, these studies provide evidence that the reimplanted autologous brain cells did not form teratoma or tumor over time.

\section{Future Perspectives}

Considering that the autologous strategy does not involve as complex ethical and/or immunosuppression concerns as in heterologous strategies, there is hope to successfully translate this approach into the clinics in the reasonably close future, for instance, in case of irreversible cerebral pathology, like brain trauma or stroke. The present animal model was aimed at reflecting mechanisms taking place after cerebral insult, such as acute lesions (vascular or trauma) leading to excitotoxicity provoking cell death (mimicked here by infusion of ibotenic acid). On a more general perspective, the present study provides additional evidence in favor of a strategy aimed at recruiting endogenous stem cells or progenitor cells, associated in synergy with exogenous stems cell actions, as recently advocated. ${ }^{12}$

\section{Disclosure}

This work was supported by the Swiss National Science Foundation, grants 31 61857.00, 310000-110005, and 31003A-132465 (to E.M.R.), 3100A0-103924 (to J.B.), the Novartis Foundation; the National Centre of Competence in Research on "Neural plasticity and repair" and the Christopher Reeves Foundation (Springfield, NJ). The authors have no personal financial or institutional interest in any of the drugs, materials, or devices described in this article.

\section{REFERENCES}

1. Lindvall O, Kokaia Z, Martinez-Serrano A. Stem cell therapy for human neurodegenerative disorders-how to make it work. Nat Med. 2004;10(Suppl):S42-S50.

2. Rosser AE, Zietlow R, Dunnett SB. Stem cell transplantation for neurodegenerative diseases. Curr Opin Neurol. 2007;20(6):688-692.

3. Imitola J. Prospects for neural stem cell-based therapies for neurological diseases. Neurotherapeutics. 2007;4(4):701-714.

4. Ormerod BK, Palmer TD, Caldwell. MA. Neurodegeneration and cell replacement. Philos Trans R Soc Lond B Biol Sci. 2008;363(1489):153-170.

5. McKay R, Kittappa R. Will stem cell biology generate new therapies for Parkinson's disease? Neuron. 2008;58(5):659-661.

6. Kondziolka D, Wechsler L. Stroke repair with cell transplantation: neuronal cells, neuroprogenitor cells, and stem cells. Neurosurg Focus. 2008;24(3-4):E13.

7. Williams BA, Keating A. Cell therapy for age-related disorders: myocardial infarction and stroke-a mini-review. Gerontology. 2008;54(5):300-311.

8. Hess DC, Borlongan CV. Stem cells and neurological diseases. Cell Prolif. 2008;41(Suppl 1):94-114.

9. Bacigaluppi M, Pluchino S, Martino G, Kilic E, Hermann DM. Neural stem/ precursor cells for the treatment of ischemic stroke. J Neurol Sci. 2008;265(1-2):73-77.

10. Locatelli F, Bersano A, Ballabio E, et al. Stem cell therapy in stroke. Cell Mol Life Sci. 2009;66(5):757-772.

11. Preynat-Sauve O, Burkhard PR, Villard J, et al. Pluripotent stem cells as new drugs? The example of Parkinson's disease. Int J Pharm. 2009;381(2):113-121.

12. Madhavan L, Collier TJ. A synergistic approach for neural repair: cell transplantation and induction of endogenous precursor cell activity. Neuropharmacology. 2009;58(6):835-844.

13. Brunet JF, Pellerin L, Arsenijevic Y, Magistretti P, Villemure JG. A novel method for in vitro production of human glial-like cells from neurosurgical resection tissue. Lab Invest. 2002;82(6):809-812.

14. Brunet JF, Pellerin L, Magistretti P, Villemure JG. Cryopreservation of human brain tissue allowing timely production of viable adult human brain cells for autologous transplantation. Cryobiology. 2003;47(2):179-183.

15. Brunet JF, Rouiller E, Wannier T, Villemure JG, Bloch J. Primate adult brain cell autotransplantation, a new tool for brain repair? Ex Neurology. 2005;196(1):195-198.

16. Courtine G, Bunge MB, Fawcett JW, et al. Can experiments in nonhuman primates expedite the translation of treatments for spinal cord injury in humans? Nat Med. 2007;13(5):561-566.

17. Capitanio JP, Emborg ME Contributions of non-human primates to neuroscience research. Lancet. 2008;371(9618):1126-1135.

18. Liu Y, Rouiller EM. Mechanisms of recovery of dexterity following unilateral lesion of the sensorimotor cortex in adult monkeys. Exp Brain Res. 1999;128(1-2):149-159.

19. Murata $Y$, Higo N, Oishi T, et al. Effects of motor training on the recovery of manual dexterity after primary motor cortex lesion in macaque monkeys. $J$ Neurophysiol. 2008;99(2):773-786.

20. Darling WG, Pizzimenti MA, Rotella DL, et al. Volumetric effects of motor cortex injury on recovery of dexterous movements. Exp Neurol. 2009;220(1): 90-108. 
21. Wyss A, Bloch J, Mir A, Schwab ME, Rouiller EM, Belhaj-Saif A. Enhancement of hand dexterity recovery following a lesion of the primary motor cortex (M1) with anti-Nogo-A antibody treatment: electrophysiological data in monkeys. Abstract FENS Meeting, July 12 to 16 , 2008, Geneva, Switzerland.

22. Kaeser M, Wyss AF, Bashir S, et al. Effects of unilateral motor cortex lesion on ipsilesional hand's reach and grasp performance in monkeys: relationship with recovery in the contralesional hand. J. Neurophysiol. 2010;103(3):1630-1645.

23. Freund P, Schmidlin E, Wannier T, et al. Anti-Nogo-A antibody treatment promotes recovery of manual dexterity after unilateral cervical lesion in adult primates-re-examination and extension of behavioral data. Eur $J$ Neurosci. 2009;29(5):983-996.

24. Brinkman J, Kuypers HGJM. Cerebral control of contralateral and ipsilateral arm, hand and finger movements in the split-brain rhesus monkey. Brain. 1973;96(4):653-674.

25. Brinkman C. Supplementary motor area of the monkey's cerebral cortex: shortand long-term deficits after unilateral ablation and the effects of subsequent callosal section. J Neurosci. 1984;4(4):918-929.

26. Nishimura Y, Onoe H, Morichika Y, Perfiliev S, Tsukada H, Isa, T. Timedependent central compensatory mechanisms of finger dexterity after spinal cord injury. Science. 2007;318(5853):1150-1155.

27. Schmidlin E, Wannier T, Bloch J, Rouiller EM. Progressive plastic changes in the hand representation of the primary motor cortex parallel incomplete recovery from a unilateral section of the corticospinal tract at cervical level in monkeys. Brain Res. 2004;1017(1-2):172-183.

28. Brunet JF, Redmond DE Jr, Bloch J. Primate adult brain cell autotransplantation, a pilot study in asymptomatic MPTP-trained monkeys. Cell Transpl. 2009b;18(7): 787-799.

29. Wannier T, Schmidlin E, Bloch J, Rouiller EM. A unilateral section of the corticospinal tract at cervical level in primates does not lead to measurable cell loss in motor cortex. J Neurotrauma. 2005;22(6):703-717.

30. Koch P, Opitz T, Steinbeck JA, Ladewig J, Brustle O. A rosette-type, self-renewing human ES cell-derived neural stem cell with potential for in vitro instruction and synaptic integration. Proc Natl Acad Sci U S A. 2009;106(9):3225-3230.

31. Horesh D, Sapir T, Francis F, et al. Doublecortin, a stabilizer of microtubules. Hum Mol Genet. 1999;8(9):1599-1610.

32. Zhang ZG, Chopp M. Neurorestorative therapies for stroke: underlying mechanisms and translation to the clinic. Lancet Neurol. 2009;8(5):491-500.

33. Frost SB, Barbay S, Friel KM, Plautz EJ, Nudo RJ. Reorganization of remote cortical regions after ischemic brain injury: a potential substrate for stroke recovery. J Neurophysiol. 2003;89(6):3205-3214.

34. Plautz EJ, Barbay S, Frost SB, et al. Post-infarct cortical plasticity and behavioral recovery using concurrent cortical stimulation and rehabilitative training: a feasibility study in primates. Neurol Res. 2003;25(8):801-810.

35. Dancause N, Barbay S, Frost SB, et al. Effects of small ischemic lesions in the primary motor cortex on neurophysiological organization in ventral premotor cortex. J Neurophysiol. 2006;96(6):3506-3511.

36. McNeal DW, Darling WG, Ge J, et al. Selective long-term reorganization of the corticospinal projection from the supplementary motor cortex following recovery from lateral motor cortex injury. J Comp Neurol. 2010;518(5):586-621.

37. Dancause N, Barbay S, Frost SB, et al. Extensive cortical rewiring after brain injury. J Neurosci. 2005;25(44):10167-10179.

38. Freund P, Schmidlin E, Wannier T, et al. Nogo-A-specific antibody treatment enhances sprouting and functional recovery after cervical lesion in adult primates. Nat Med. 2006;12(7):790-792.

39. Roitberg BZ, Mangubat E, Chen EY, et al. Survival and early differentiation of human neural stem cells transplanted in a nonhuman primate model of stroke. J Neurosurg. 2006;105(1):96-102.

40. Takagi Y, Takahashi J, Saiki H, et al. Dopaminergic neurons generated from monkey embryonic stem cells function in a Parkinson primate model. J Clin Invest. 2005;115(1):102-109.

41. Brunet JF, Redmond DE Jr, Bloch J Primate adult brain cell autotransplantation improves the Parkinson score in a MPTP parkinsonian monkey model. Abstract. Society for Neuroscience October 17 to 21, 2009, Chicago, IL.

\section{Acknowledgments}

The authors wish to thank the technical assistance of Laurence Grollimund, Catherine Pythoud, Véronique Moret, Christine Roulin, Françoise Tinguely and Christiane Marti (histology and behavioral evaluations), Josef Corpataux, Laurent
Bossy, Bernard Bapst and Bernard Morandi (animal house keeping), André Gaillard (mechanics), Bernard Aebischer (electronics), Laurent Monney (informatics). Thanks are due to Dr T. Wannier, Dr S. Bashir, and Dr E. Schmidlin for their support and advice in the course of the experiments. Julie Savidan contributed to the assessment of the volume of cortical lesion. 
and the reduction of infarct volume related more to the neuroprotective effects rather than the formation of a new network between host neurons and the implanted cells. ${ }^{4}$ Endogenous neurogenesis has been observed in response to ischemic injury, and can be enhanced via transplants or infusion of appropriate cytokines. Enriched housing and voluntary running exercise enhanced migration of transplanted stem cells toward the region of injury after stroke and trends to the increase of survival of stem cells. ${ }^{5}$ Thus the ability of transplanted stem cells in promoting recovery can be augmented by environmental factors such as rehabilitation. For maximal functional recovery, regenerative therapy will need to incorporate multidisciplinary approaches, which may include cell replacement, trophic support, protection from oxidative stress, and enhanced endogenous neuronal stem cells response. The loss of appropriate guidance cues and supporting architecture in the infarct cavity will likely impede the restoration of lost circuitry. Biologically compatible nanofiber scaffolds may provide the structure needed, ${ }^{6}$ but getting grafted neurons to survive and make functional synapses as replacements to lost neurons is still a dream.

Before embracing clinical applications, several essential precautions must be suitably addressed. For example, the regenerative potentials of adult stem cells decline with age, and stem cells isolated from aged patients may retain dysfunctional characteristics. Functional recovery from stroke reaches a maximum level by 3 to 6 months after onset, and no further recovery occurs beyond this time. The time course for therapeutic intervention still needs to be worked out. Are the available autologous cells appropriate for therapeutic application in stroke? What are the optimal injection routes and cell doses? Success in future clinical trials will depend on careful investigation at the experimental level, to allow us to understand not only the clinical practicalities of stem cell use, but also the underlying biological principles involved. Furthermore, stimulation of endogenous adult stem cell-mediated repair mechanisms in the brain might offer new avenues for stroke therapy without the necessity of transplantation. Thus, important scientific issues need to be addressed to advance our understanding of the molecular mechanisms underlying the critical steps in cell-based repair to allow the introduction of these experimental techniques into clinical practice.

Roy Bakay Chicago, Illinois

1. Urbaniak Hunter K, Yarbrough C, Ciacci J. Stem cells in the treatment of stroke. Adv Exp Med Biol. 2010;671:105-116.

2. Burns TC, Verfaillie CM, Low WC. Stem cells for ischemic brain injury: a critical review. J Comp Neurol. 2009;515(1):125-144.

3. Kaeser M, Wyss AF, Bashir S, et al. Effects of unilateral motor cortex lesion on ipsilesional hand's reach and grasp performance in monkeys: relationship with recovery in the contralesional hand. J Neurophysiol. 2010;103(3):1630-1645.

4. Koh SH, Kim KS, Choi MR, et al. Implantation of human umbilical cord-derived mesenchymal stem cells as a neuroprotective therapy for ischemic stroke in rats. Brain Res. 2008;1229:233-248.

5. Hicks AU, Hewlett K, Windle V, et al. Enriched environment enhances transplanted subventricular zone stem cell migration and functional recovery after stroke. Neuroscience. 2007;146(1):31-40.

6. Kubinova S, Sykova E. Nanotechnology for treatment of stroke and spinal cord injury. Nanomedicine (Lond), 2010;5(1):99-108.

\section{Follow NEUROSURGERY ${ }^{\circledR}$ on YouTube}
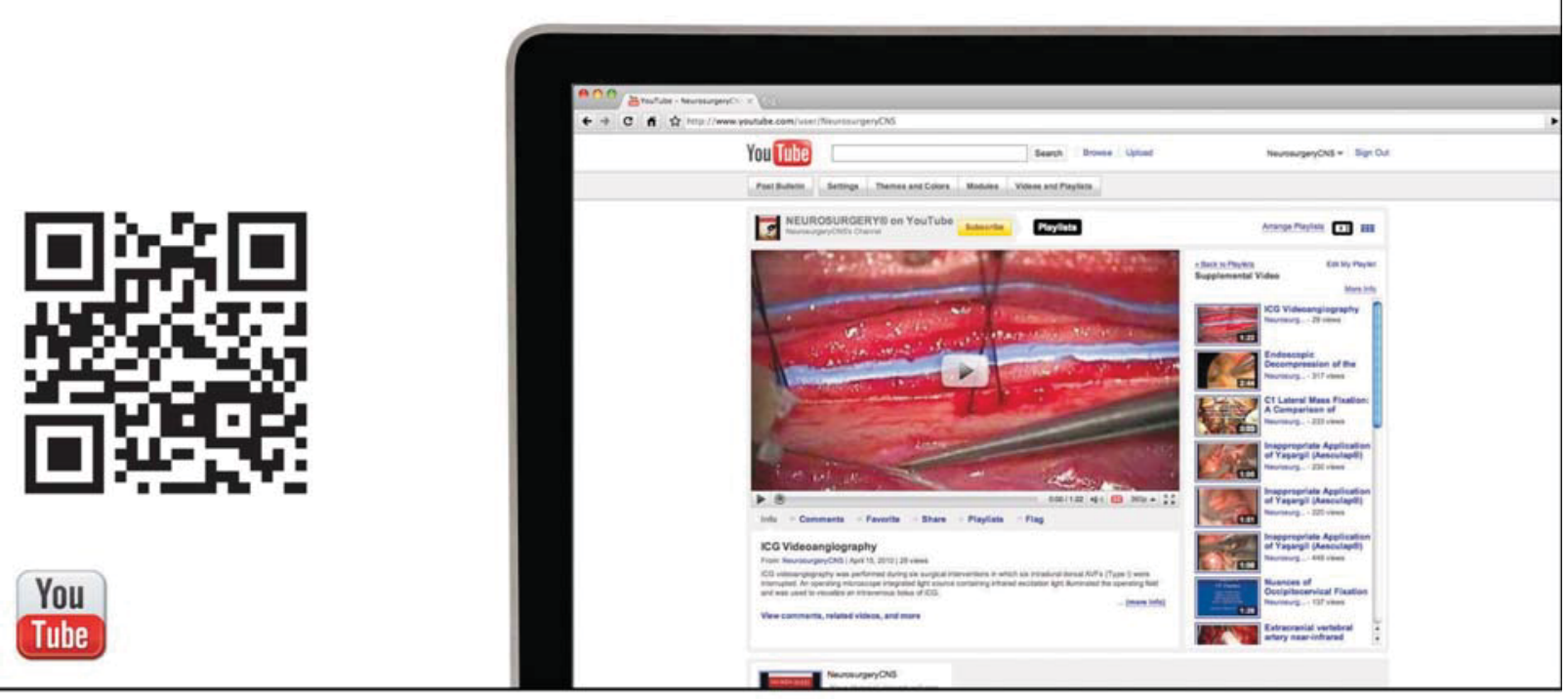

Follow NEUROSURGERY` at http://www.youtube.com/user/NeurosurgeryCNS 